\title{
Exhaled air speed measurements of respiratory air flow, generated by ten different human subjects, under uncontrolled conditions
}

\author{
Martin Ivanov ${ }^{1}$ \\ ${ }^{1}$ Technical University - Sofia, FPEPM, Department:” Hydroaerodynamics and Hydraulic Machines”, Sofia 1000, Bulgaria
}

\begin{abstract}
The presented study reveals a multi-point experimental measurement of the airflow speed, generated in the exhalation phase from the human respiratory cycle, based on data from 10 real human subjects. The results obtained demonstrate the exceptional irregularity and the transient character of the human respiratory cycle, in terms of measured speed at different points of man-generated exhalation air flow. The presented exponential trend lines show as expected, that there is a reduction in the exhalation speed, with the increase of the distance from the nose.

The summary analysis shows that at $10 \mathrm{~cm}$ distance from the nose the maximum as well as the average measured speeds are higher than the one, measured at $5 \mathrm{~cm}$ distance. This suggests that due to the physiology of the human nose, $5 \mathrm{~cm}$ distance is not enough to characterize the fully developed exhalation jet flow. It is suggested that at this distance the exhalation jets are still two, issuing from each nostril, with different flowrate ratio.

The obtained flow characteristics can be used to compare, validate and verify data from conducted numerical studies of the breathing process, via virtual models or real breathing thermal mannikins.
\end{abstract}

\section{Introduction}

Breathing is the most essential activity of the human body and practically people cannot exist without breathing $[1,2,3]$. By the exchange of inhaled and exhaled air, the human organism is decontaminated from the harmful substances and gases, generated by the metabolism $[3,4,5]$. An extremely important part of the respiratory function is also the removal of carbon dioxide from the lungs, and thus purifying the blood system. The respiratory cycle in humans is characterized by the air flow from and out of the lungs. Healthy adults usually breathe in and out between 10 and 15 times per minute, depending on the degree of their physical activity. Children inhale and exhale between 18 and 20 times per minute $[3,5]$. Also, about 0.6 liters of air is exchanged between the lungs and the environment during a normal breathing cycle. In summary, this corresponds to inhaling and exhaling of more than 12900 liters of air per day.

On the other hand, the processes of inhaling and exhaling in humans are highly transient in nature and extremely difficult to study. The physiology of the individuals is largely the major cause of this nonstationarity. Large number of studies have been documented over the past years, related to the human respiratory cycle $[6,7,8]$. Taking into account the physiological differences in humans, most of these studies used thermal manikins, simulating respiration and other physiological processes $[9,10,11]$. However, the physical replacement of the human being in the experimental settings leads to compromising of the obtained results. It is also common practice in similar numerical studies, to validate numerical results again with experimental data from thermal manikins $[8,12]$. Thus, the consistency with the real physical phenomena in the "human-environment" system is again compromised.

The foregoing shows the necessity for experimental studies in the presented area, in which the respiratory air flow is generated by a real human. The exhalation phase is of particular interest, as it carries out the removal of the generated by the metabolism harmful gases into the environment. The maximum air flow distribution at exhalation principally defines the "breathing zone" of a person. Also, the airflow during exhalation has a significant impact on the convective boundary layer around the human body.

Performing experimental measurements of the respiratory airflow parameters, would assist the validation and verification of the numerical simulation studies conducted in the presented area. However, due to the strong physiological differences between people and the existing process unsteadiness, carrying out such type of research is a difficult and multidisciplinary task. 


\section{Objective of the study}

The aim of the presented study is to perform a multipoint experimental measurement of the air flow speed, generated during the exhalation phase of the respiratory cycle in humans.

\section{Methods}

The main method used is based on experimental measurement of the physical characteristics of an airflow, generated in the exhalation phase of the human respiration cycle. The air flow is generated by a real human being, under uncontrolled conditions, reflecting the real physical phenomena. The experimental study was not conducted in a controlled environment (climatic chamber) but in a fully realistic work environment. The selection of the participants is entirely random.

The measured breathing air flow parameters are speed and temperature. The measurement points were pre-established in an experimental visualization of the exhalation flow by a cigarette smoke. To facilitate the participants and to make the results more precise, the measurements are limited to 5 points in one plane, in the assumed centre of the air jet. Each point is located in 5 $\mathrm{cm}$ horizontal and vertical displacement from the nose, starting at $5 \mathrm{~cm}$ and ending at $25 \mathrm{~cm}$ distance.

For additional characterization of the exhalation flow interaction, with the convective boundary layer around the human body, the surface temperature of the participants was measured in parallel, by means of an infrared thermal imaging camera. The measurement is concentrated on the top of the human body (the torso, head and arms above the legs), where the thermal convective layer expands and interacts with the breathing zone. Again, in order to facilitate and refine the data obtained, the infrared images are made at the beginning and at the end of the measurement of the exhalation flow speed, which is within an interval of 3 minutes. It is known that, the surface temperature distribution over the human body significantly influences the thickness of the convective boundary layer. But also, this will affect the speed and the velocity of the air flow during exhalation and in turn will lead to a change in the parameters of the human breathing zone area, from ergonomic point of view.

Last but not least, the participants themselves were also characterized by a short paper-based survey, prior to the experimental measurements. The questionnaires contain a set of questions about the physical characteristics of the individual participant, such as age, gender, height, weight, and current health status.

\section{Experimental set-up and instrumentation}

The experimental set-up was established in Hall 2126, located on the first floor of Building 2 of the Technical University - Sofia, Bulgaria. The hall has dimensions: 7 $\mathrm{m} \times 15 \mathrm{~m} \times 4 \mathrm{~m}$, which implies the presence of $420 \mathrm{~m}^{3}$ of air. The room is initially kept almost empty and has three outer walls with north-east-south exposure. The north and south façades are fully glazed.

Taking into account the short measuring interval, it is considered that the size of the room is sufficient to adequately conduct the proposed study, without the environment to significantly affect the obtained results. However, such experimental research should be conducted in specialized climatic chambers, under controlled microclimate parameters.

During the measurements, all windows and doors were closed and only one participant and one operator were present in the room, to minimize the impact over the indoor environment. The homogeneity of the room parameters was not controlled, only the air temperature during the measurements is observed and partially analysed. Data on outdoor weather conditions were provided by the National Institute of Meteorology and Hydrology at the Bulgarian Academy of Sciences. The experimental study was conducted on 04. July. 2018 and according to the average data, the outside air temperature is in the range $28 \div 29^{\circ} \mathrm{C}$, the atmospheric pressure is close to the average for the month in Sofia, the humidity of the outside air is $53 \%$ and there is a mild to moderate wind at the speed of $2.5 \mathrm{~m} / \mathrm{sec}$. The room temperature was in the range $23 \div 24{ }^{\circ} \mathrm{C}$.

Simplified scheme (non-scaled) of the experimental set-up, with the positioning of the object and the measuring devices, is presented in Figure 1. The use of a real person, to generate the respiratory air flow, significantly limits the possible techniques for exhalation flow speed and velocity measurements. Advanced precision methods based on laser technologies such as PIV (Particle Image Velocimetry) and LDA (LaserDoppler Anemometry) are dangerous to human health and are obviously not applicable in this case. That is why, a "Testo 435" hot-wire anemometer is used to measure the exhalation air speed. It has a built-in logger and a probe, allowing measurements in the range $0 \div 20$ $\mathrm{m} / \mathrm{sec}$ with a step of $0.01 \mathrm{~m} / \mathrm{sec}$, and accuracy of $\pm 4 \%$. But, it should be mentioned that this type of device does not account for the direction of the flow, i.e. the flow velocity components in $\mathrm{x}, \mathrm{y}$ and $\mathrm{z}$ direction. The hotwire anemometer is mounted on a tripod, which allows manual movement in horizontal and vertical directions. The precise positioning is achieved by using additional markers. The tripod is positioned at $70 \mathrm{~cm}$ away of the participant, outside the breathing zone, so as not to interfere with the physical measurements. The speed records were performed for every second, throughout the entire experimental period.

The infrared thermal imaging camera, used to measure the surface temperature of the participants, is FLIR C3. It allows measurements at 4800 points in the temperature range $-10 \div 150{ }^{\circ} \mathrm{C}$, with accuracy $\pm 2 \%$. The camera is mounted on its own tripod at $2 \mathrm{~m}$ distance from the participant, again outside the breathing zone, so as not to disturb the physical measurements of the air flow speed. The focal length is adjusted so that the 


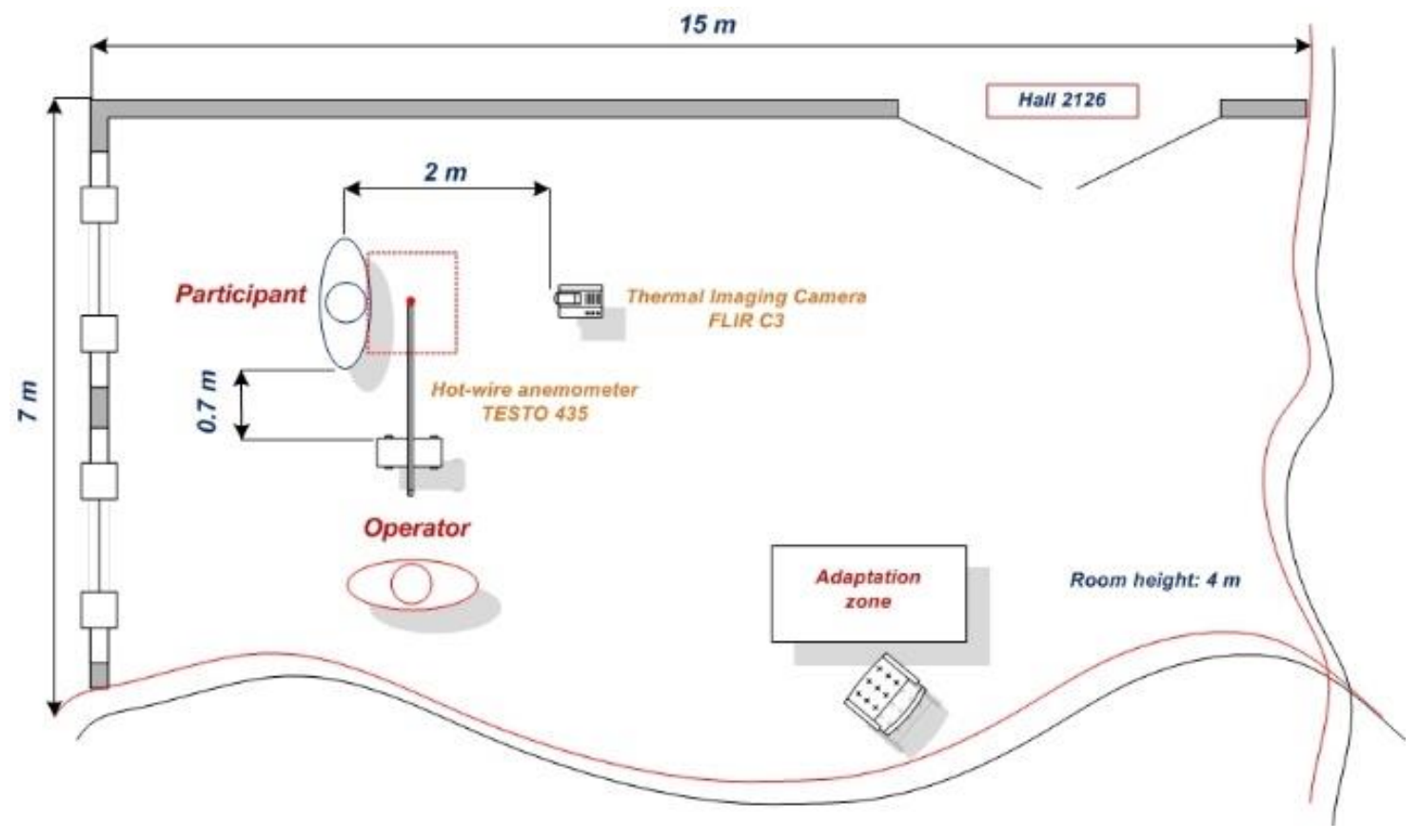

Fig. 1. Simplified scheme of the experimental set-up

measuring points cover the top of the body of the participant. There are 5 distinct measuring zones "Nose", "Neck", "Shoulders", "Chest" and "Abdominal area".

For the characterization of the participants themselves, the abovementioned simplified questionnaires were used. They contain a set of questions concerning the participants' physiological characteristics and current health status. These questionnaires were filled in by each participant, prior to the physical measurements. This time is also used for adaptation of the participant to the room's thermal environment. The questionnaires are important tool in the presented study and their information may help to refine the achieved results.

\section{Experimental procedure and participants}

As mentioned, the adequate measurement of the exhalation airflow speed and velocity, generated by human being, is quite complex task. On one hand, this is due to the non-stationarity of the respiratory cycle in humans and due to the physiological differences between the individuals. But, on the other hand, it is a serious challenge for the participants themselves to stay motionless for a long time in the same position, breathing evenly. That is why, the experimental measurements are performed in three minutes intervals, or cover a total of 180 seconds. This interval guarantees at least 45 inhalations and exhalations, depending on the current state of the participant, averaging at the number of 60 .

The measurements were performed at 5 different distances from the nose, in one plane (horizontal and vertical displacement of $5 \mathrm{~cm}, 10 \mathrm{~cm}, 15 \mathrm{~cm}, 20 \mathrm{~cm}$ and
$25 \mathrm{~cm}$ ). This makes at least 9 (averaged 15) measured speed values at a given point of the assumed centreline of the jet. At the beginning and at the end of every 3minute interval, an infrared picture of the surface temperature of each participant is made.

Summarized, the experimental study follows strictly the following procedure:

- Zeroth minute - the participant enters the room, sits in a specially organized place and completes the questionnaire for 2 minutes. This time is also meant for the normalization of breathing, as well as for adaptation with the thermal environment.

- Second minute - the participant takes the place specified for the measurements. The operator adjusts the hot-wire anemometer, according to the individual height, and sets the starting point at $5 \mathrm{~cm}$ from the nose.

- Fifth minute - starting of the exhalation speed measurements and first infrared picture. At every 36 seconds, the operator manually moves the hot-wire anemometer probe, at $5 \mathrm{~cm}$ in a horizontal and vertical direction, to reach a $25 \mathrm{~cm}$ distance.

- Eighth minute - end of the exhalation speed measurements and second infrared picture.

- Ninth minute - the participant leaves the room, which due to its large volume is assumed to reach a steady state conditions within 10 minutes.

In total of 10 participants took part as volunteers in the experimental study, selected among employees and students of the Technical University - Sofia. Among them, there were three men and seven women. The average age of the participants is 40 years and their average height is $175 \mathrm{~cm}$, and average weight is $70 \mathrm{~kg}$. Three of them are smokers. According to the survey data, none of the respondents reported any respiratory problems, or any other type of concern before the measurements. 


\section{Results and discussion}

All results from the conducted experimental study were processed and analysed. The analysis is based on the air flow speed distribution in time, for the corresponding distances from the nose of the participants. But as it was mentioned, the hot-wire anemometer does not give information about the instantaneous velocity, i.e. the direction of the flow. That is why, additional assumption should be made, in order to distinguish between measured inhalation and exhalation flow. So, in the presented study, the maximum measured speed value at each point is considered as the recorded exhalation speed. The practice shows that, the inhalation speed is much lower than the exhalation one, in the normal breathing cycle. The interaction with the free convection boundary layer is one of the reasons for this fact.

For illustration, Figure 2 shows the measured speed values for the entire period of one randomly selected participant - Number 4 . The participant is a 42 years old female, $169 \mathrm{~cm}$ in height and $59 \mathrm{~kg}$ of weight. The participant is not a smoker, at the time of the experimental study she feels calm, does not feel her nose obstructed or irritated and does not suffer from chronic respiratory problems.

At $5 \mathrm{~cm}$ from the nose, the maximum measured air flow speed is $0.12 \mathrm{~m} / \mathrm{sec}$ (SD $0.023 \mathrm{~m} / \mathrm{sec}$ ). At a distance of $10 \mathrm{~cm}$, the maximum measured speed is $0.16 \mathrm{~m} / \mathrm{sec}$ (SD $0.031 \mathrm{~m} / \mathrm{sec}$ ), and at $15 \mathrm{~cm}$ the flow speed is 0.08 $\mathrm{m} / \mathrm{sec}(\mathrm{SD} 0.014 \mathrm{~m} / \mathrm{sec}$ ), at $20 \mathrm{~cm}$ is $0.06 \mathrm{~m} / \mathrm{sec}$ (SD $0.013 \mathrm{~m} / \mathrm{sec}$ ), and at a distance of $25 \mathrm{~cm}$ from the nose, the maximum speed is $0.07 \mathrm{~m} / \mathrm{sec}(\mathrm{SD} 0.012 \mathrm{~m} / \mathrm{sec}$ ).

The results demonstrate the expected reduction in the airflow speed value during exhalation, with the increase of the distance from the nose. But they also show the high degree of non-stationarity of the breathing process in humans. It should also be noted that some of the maximum speed values may coincide with the moment of movement of the hot-wire anemometer at a given point. Therefore, the data should be reviewed along with the standard deviation of the value in the sample interval.

In Table 1 and Figure 3 are presented the mean, maximum and minimum measured values of the speed, as well as the standard deviation for each measurement point, averaged over the ten participants in the study.

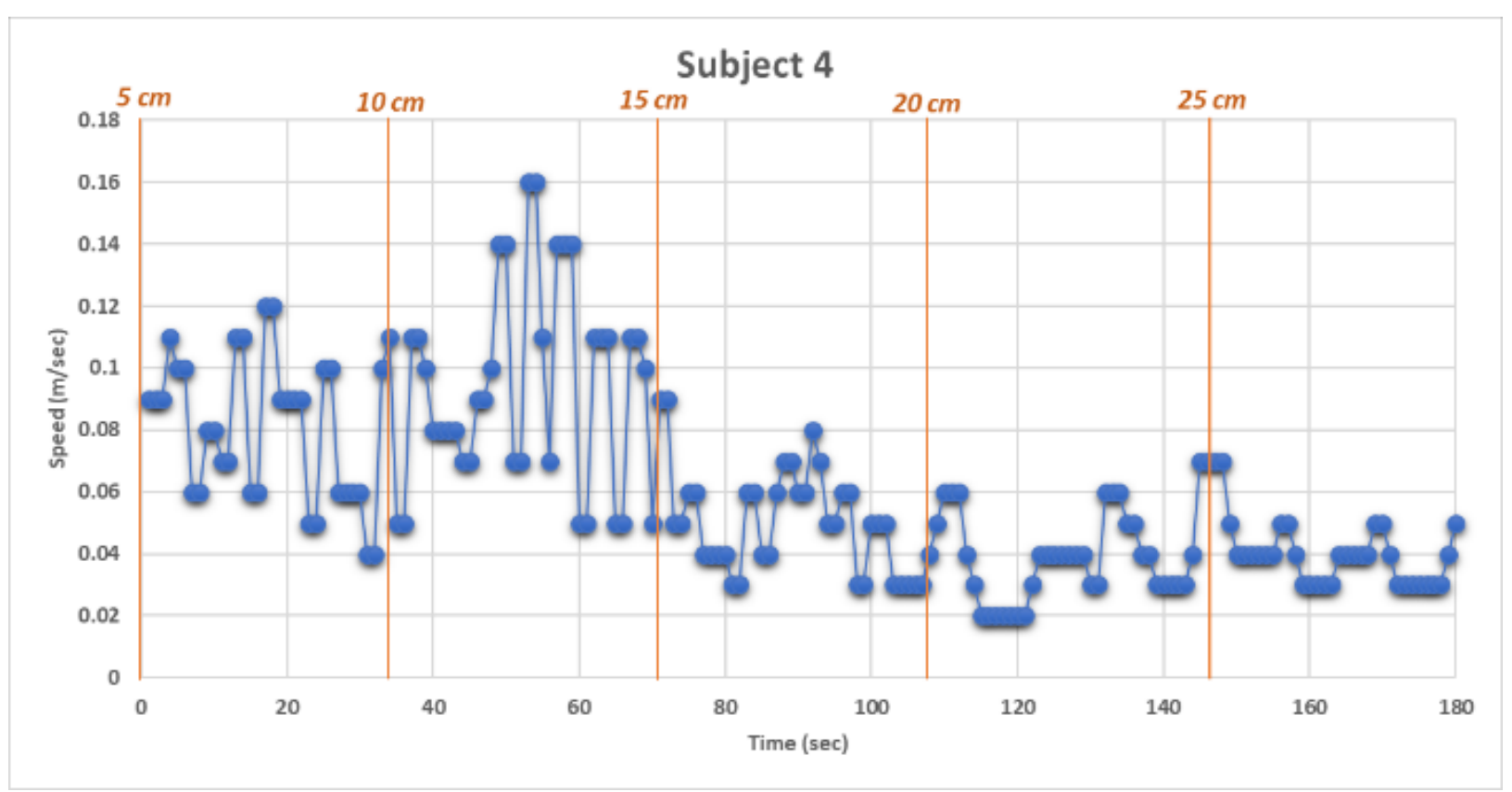

Fig. 2. Speed distribution over the entire measurement period, for participant Number 4

Table 1. Measured speeds, averaged over all ten participants.

\begin{tabular}{|c|c|c|c|c|c|c|}
\hline Distance from the nose: & $5 \mathrm{~cm}$ & $10 \mathrm{~cm}$ & $15 \mathrm{~cm}$ & $20 \mathrm{~cm}$ & $25 \mathrm{~cm}$ & \\
\hline Average speed: & 0,059972 & 0,062944 & 0,053778 & 0,044 & 0,036833 & $\mathrm{~m} / \mathrm{sec}$ \\
\hline Maximum speed: & 0,15 & 0,2 & 0,15 & 0,1 & 0,1 & $\mathbf{m} / \mathbf{s e c}$ \\
\hline Minimum speed: & 0,01 & 0,01 & 0,01 & 0,01 & 0,01 & $\mathbf{m} / \mathbf{s e c}$ \\
\hline Standard deviation: & 0,00504 & 0,008525 & 0,005252 & 0,003362 & 0,00461 & $\mathrm{~m} / \mathrm{sec}$ \\
\hline
\end{tabular}




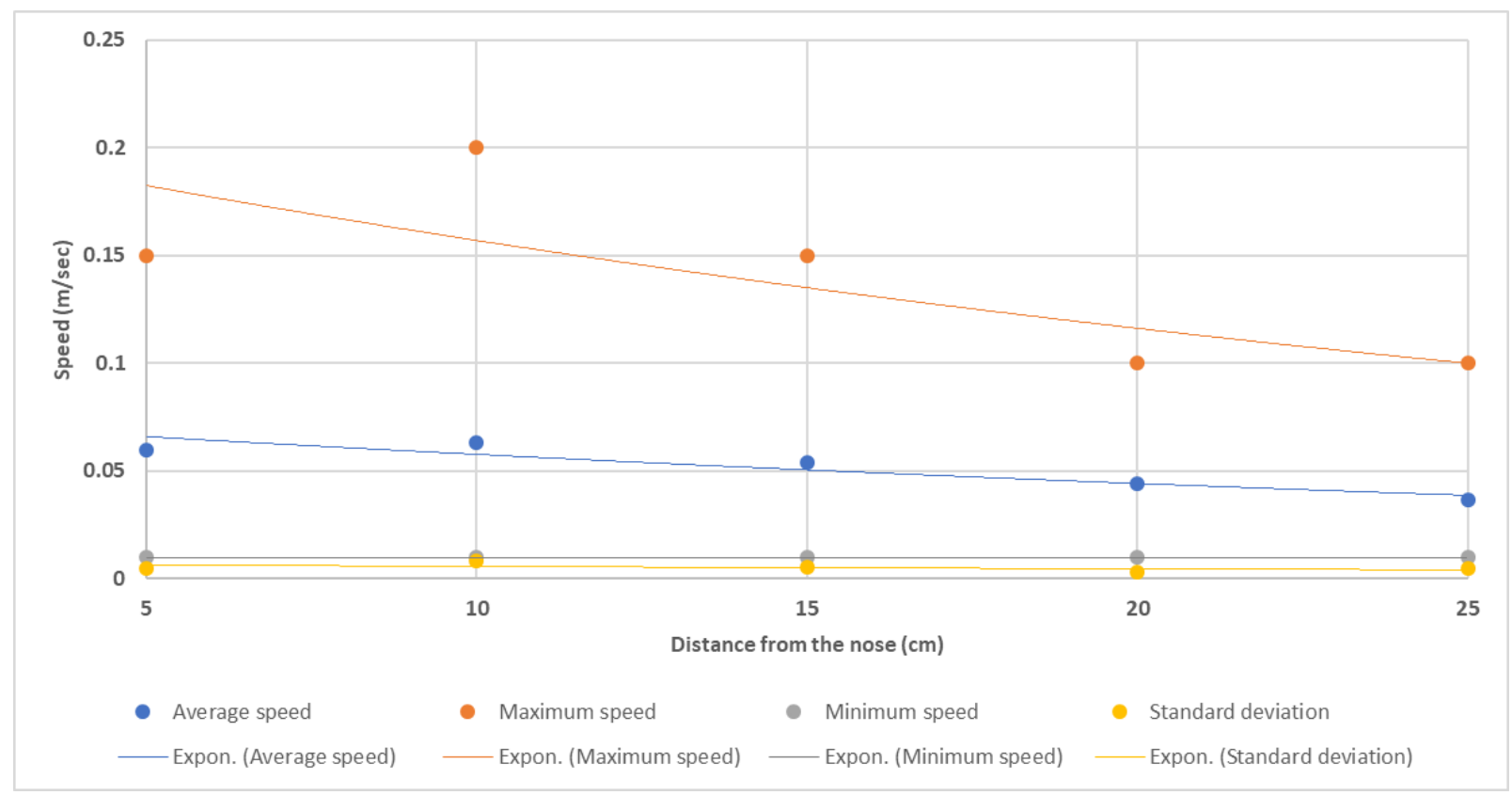

Fig. 3. Average speed distribution based on all participants, over the entire measurement period

At $5 \mathrm{~cm}$ from the nose, the average maximum flow speed is $0.15 \mathrm{~m} / \mathrm{sec}(\mathrm{SD} 0.005 \mathrm{~m} / \mathrm{sec})$. At a distance of $10 \mathrm{~cm}$, the average maximum speed is $0.2 \mathrm{~m} / \mathrm{sec}$ (SD $0.008 \mathrm{~m} / \mathrm{sec}$ ). At $15 \mathrm{~cm}$ the average maximum speed is $0.15 \mathrm{~m} / \mathrm{sec}$ (SD $0.005 \mathrm{~m} / \mathrm{sec}$ ). At a distance of $20 \mathrm{~cm}$ and $25 \mathrm{~cm}$, the average maximum speed is $0.1 \mathrm{~m} / \mathrm{sec}$ (SD $0.003 \mathrm{~m} / \mathrm{sec}$ ) and again $0.1 \mathrm{~m} / \mathrm{sec}(\mathrm{SD} 0.004 \mathrm{~m} / \mathrm{sec})$. The values of the mean minimum airflow speed are the same at the five measuring points, respectively $0.01 \mathrm{~m} / \mathrm{sec}$. The averaged mean speed values of the flow at $5 \mathrm{~cm}$ distance is $0.0599 \mathrm{~m} / \mathrm{sec}$, and at $10 \mathrm{~cm}$ is $0.0629 \mathrm{~m} / \mathrm{sec}$, at $15 \mathrm{~cm}$ is $0.0537 \mathrm{~m} / \mathrm{sec}$, at $20 \mathrm{~cm}$ is $0.044 \mathrm{~m} / \mathrm{sec}$, and at $25 \mathrm{~cm}$ is $0.0368 \mathrm{~m} / \mathrm{sec}$.

The summary analysis shows that at $10 \mathrm{~cm}$ distance from the nose the maximum as well as the average speeds are higher than the one measured at $5 \mathrm{~cm}$ distance. This suggests that due to the physiology of the human nose, $5 \mathrm{~cm}$ length is not enough for full development of the exhalation jet. At this distance the jets are still two, generated respectively by the left and right nostrils, in a different volume flowrate. This effect should be anticipated in such future studies as well as in the correct definition of the breathing zone from ergonomic point of view. The exponential lines of the trends shown in Figure 3 again illustrate the expected pattern of decreasing the exhalation speed, with the increase of the distance from the nose.

The analysed thermal images of the participants show the extremely uneven distribution of the surface temperature, unlike the similar experimental studies with thermal manikins. At the beginning of the measurement, the average surface temperature in the nose area of the participants is about $33{ }^{\circ} \mathrm{C}$, but it increases in the neck and shoulders area to over $35^{\circ} \mathrm{C}$, on average base. This increase is normal, and this temperature difference affects the velocity and the speed of the exhalation air flow, in the interaction zone with the convective flow of around the human body. This effect implies lowering the maximum flow velocity during exhalation and consequently reducing the breathing zone area.

Table 2. Surface temperature, averaged for all participants

\begin{tabular}{|r|c|c|c|c|}
\cline { 2 - 5 } \multicolumn{1}{c|}{} & \multicolumn{2}{|c|}{ Beginning of the measurements } & \multicolumn{2}{c|}{ End of the measurements } \\
\cline { 2 - 5 } & Nemperature ${ }^{\circ} \mathbf{C}$ & Tem $^{\circ} \mathbf{C}$ & Temperature ${ }^{\circ} \mathbf{C}$ & SD $^{\circ} \mathbf{C}$ \\
\hline Neck: & 33.24 & 1.259805982 & 34.17 & 0.67462 \\
\hline Shoulders: & 34.29 & 1.061916506 & 34.58 & 0.97895 \\
\hline Chest: & 32.6 & 0.843274043 & 33.25 & 1.34268 \\
\hline Abdominal area: & 31.64 & 1.195547294 & 31.95 & 1.92137 \\
\hline
\end{tabular}




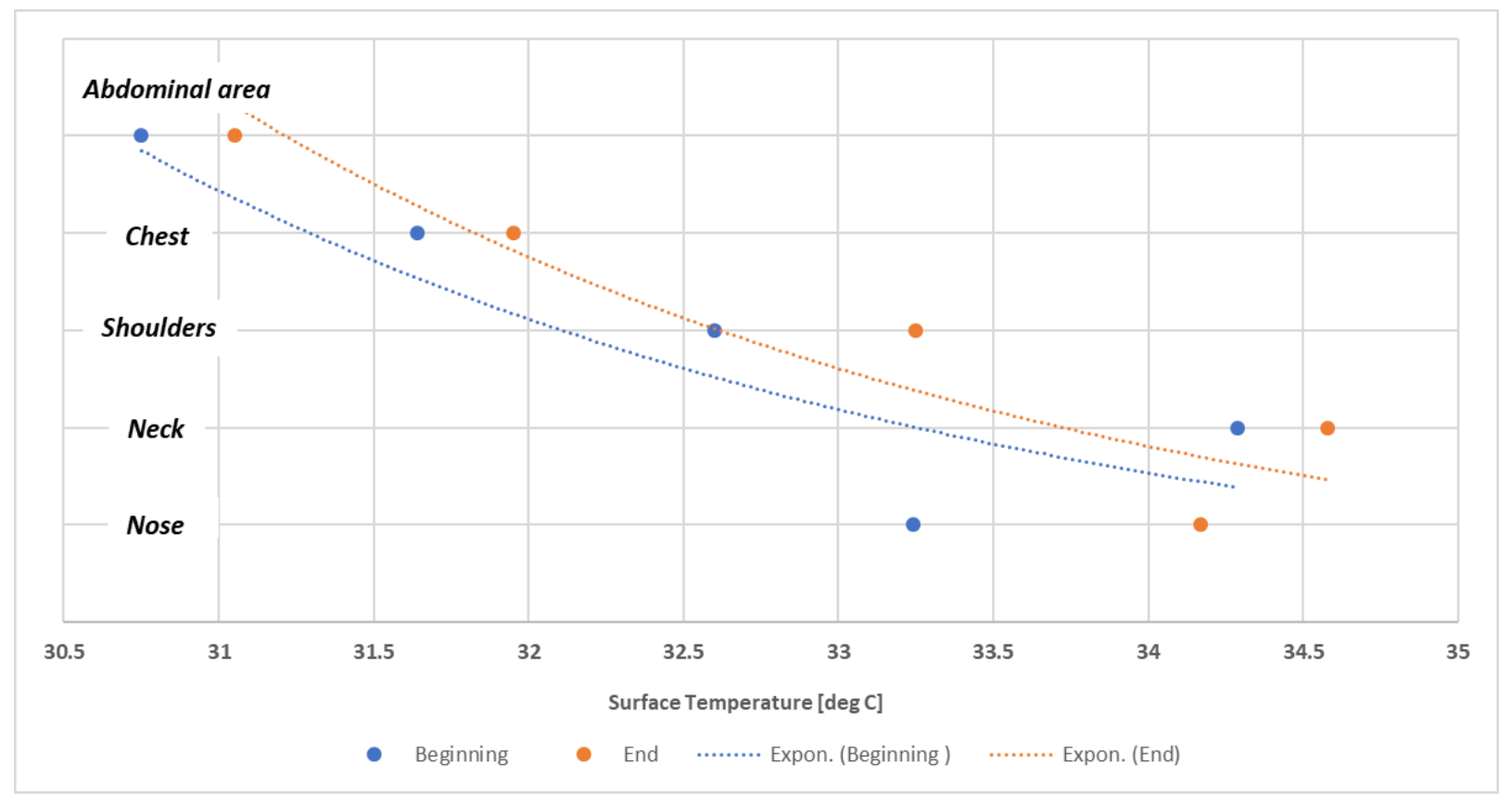

Fig. 4. Average surface temperature distribution, based on all participants

In Table 2 and Figure 4, the averaged values of the surface temperature for all participants are reported, for the beginning and at the end of the measurements. The thermal images show accurately the temperature difference between all body areas. The temperature rise between the beginning and the end of the measurement period is also noticeable. This is an unexpected result, considering the short measuring interval.

The average recorded surface temperature in the neck area is highest at both the beginning and the end of the measurements. The exponential lines in Figure 4 demonstrate the overall tendency of increasing the surface temperature towards the areas near the breathing zone - around the nose, neck and shoulders.

The high degree of non-stationarity of the breathing process in humans is largely confirmed by the so organized experiment. However, there is a need for several improvements of the experimental set-up and procedure. It is recommended an automated simultaneous multipoint measurement, with minimal operator involvement. The experiments should be performed under controlled environmental conditions, correspondingly in climatic chambers. Also, a larger number of participants is needed to collect more experimental data for adequate statistical analysis of the information.

Last but not least, the use of hot-wire anemometer in this experimental study inevitably abandons the measurement of the three components of the velocity vector. That is why, the directions of the inhalation and exhalation flows are simply approximated, based on the received speed values, described above. More advanced measurement technique is need for such experimental studies. However, this will be quite challenging, because real human beings are used, for the breathing flow generation. As is was mentioned, replacing the humans with thermal manikins will inevitable compromise the achieved results, especially if validation data is need for numerical studies.

\section{Conclusions}

A multi-point experimental measurement of the airflow speed, generated during the exhalation phase of the human respiratory cycle, was performed. The air flow was generated by real human beings and the analyses are based over the measurements from 10 randomly selected participants.

The results obtained demonstrate the exceptional irregularity of the human respiratory cycle, both in terms of measured exhalation speed and measured body surface temperature. The presented exponential trend lines show the expected reduction in the exhalation speed, with the increase of the distance from the nose.

The summary analysis shows that at $10 \mathrm{~cm}$ distance from the nose the maximum as well as the average speeds are higher than the one at $5 \mathrm{~cm}$ distance. This suggests that, due to the physiology of the human nose, 5 $\mathrm{cm}$ distance is not enough to fully develop the exhalation jet flow.

The temperature measured in the nose area is lower, compared to the area of the neck and the shoulders of the participants. This effect is normal, and this temperature difference affects the speed of the exhalation air flow in the mixing zone with the convective boundary layer flow, around the body. This effect implies lowering the maximum flow speed at exhalation and consequently reducing the breathing zone area.

It is observed an increase of the surface temperature, between the beginning and the end of the measurement period, in all body zones. This is an unexpected result, considering the short measurement interval, and should be further analysed. Reasons will also be sought in the organization of the experiment itself. 
The obtained flow characteristics can be used to compare, validate and verify data from conducted numerical studies of the breathing process, via virtual models or real breathing thermal mannikins.

\section{Acknowledgements}

The presented study is supported by "RDS" at TU-Sofia, as part of the activities under the "Perspective leaders" project, with Contract № 191ПР0007-02, entitled: "Numerical assessment of the convective flow characteristics around improved polygonal thermal manikin, based on the Computational Fluid Dynamics' methods".

\section{References}

1. A. Bogdan, B. Koelblen, M. Chludzinska, "Influence of a breathing process on the perception of the thermal environment using personalised ventilation", J. Build. Environ., Elsevier, Vol. 96, pp. 80-90 (2016)

2. B. BuSha, G. Banis, "A stochastic and integrative model of breathing”, J. Respir. Physiol. Neurobiol., Elsevier, Vol. 237, pp. 51-56 (2017)

3. M. Ivanov, S. Mijorski, "CFD modelling of flow interaction in the breathing zone of a virtual thermal manikin", J. Energy Procedia, Vol. 112, pp. 240251, ISSN: 1876-6102, Elsevier (2017)

4. J. Laverge, M. Spilak, A. Novoselac, "Experimental assessment of the inhalation zone of standing, sitting and sleeping persons", J. Build. Environ., Elsevier, Vol. 82, pp. 258-266 (2014)

5. T. Sbrana, A. Landib, G. Catapano, "Innovative model to simulate exhalation phase in human respiratory system", J. Comput. Methods. Programs. Biomed., Elsevier, Vol. 104, pp. 300-305 (2011)

6. L. Feng, S. Yao, H. Sun, N. Jiang, J. Liu, “TR-PIV measurement of exhaled flow using a breathing thermal manikin", J. Build. Environ., Elsevier, Vol. 94, pp. 683-693 (2015)

7. N. Jiang, S. Yao, L. Feng, H. Sun, J. Liu, "Experimental study on flow behaviour of breathing activity produced by a thermal manikin", J. Build. Environ., Elsevier, Vol. 123, pp. 200-210 (2017)

8. C. Li, J. Jiang, H. Dong, K. Zhao, "Computational modelling and validation of human nasal airflow under various breathing conditions", J. Biomech., Elsevier, Vol. 64, pp. 59-68 (2017)

9. M. Mhetre, H. Abhyankar, "Human exhaled air energy harvesting with specific reference to PVDF film", J. Eng. Sci. Technol., Elsevier, Vol. 20, pp. 332-339 (2017)

10. J. Villafruela, I. Olmedo, M. Ruiz de Adana, C. Méndez, P.V. Nielsen, "CFD analysis of the human exhalation flow using different boundary conditions and ventilation strategies", J. Build. Environ., Elsevier, Vol. 62, pp. 191-200 (2013)
11. S. Yoo, K. Ito, "Assessment of transient inhalation exposure using in silico human model integrated with PBPK-CFD hybrid analysis", J. Sustain. Cities Soc., Elsevier, Vol. 40, pp. 317-325 (2018)

12. T. Zhang, S. Yin, S. Wang, "Quantify impacted scope of human expired air under different head postures and varying exhalation rates", J. Build. Environ., Elsevier, Vol. 46, pp. 1928-1936 (2011) 